\title{
An inter-laboratory comparison of low-level measurements in ground-level aerosol monitoring
}

\author{
H. Wershofen ${ }^{\mathrm{a}, *}$, J. Bieringer ${ }^{\mathrm{b}}$, S. Frenzel ${ }^{\mathrm{c}}$, G. Kanisch ${ }^{\mathrm{d}}$, C. Katzlberger ${ }^{\mathrm{e}}$, \\ Th. Steinkopff ${ }^{\mathrm{f}}$, J. Tschiersch ${ }^{\mathrm{g}}$, H. Völkle ${ }^{\mathrm{h}}$ \\ ${ }^{a}$ Physikalisch-Technische Bundesanstalt, WG 6.12 Environmental Radioactivity, Bundesallee 100, 38116 Braunschweig, Germany \\ ${ }^{\mathrm{b}}$ Federal Office for Radiation Protection (BfS), SW 2.5 Atmospheric Radioactivity and Trace Analysis, Willy-Brandt-Street 5, 38226 Salzgitter, Germany \\ ${ }^{\mathrm{c}}$ Radiochem-Consult Stefan Frenzel, Kiefernweg 5, 63263 Neu-Isenburg, Germany \\ ${ }^{\mathrm{d}}$ Federal Research Centre for Fisheries, Institute for Fishery Ecology, Palmaille 9, 22767 Hamburg, Germany \\ ${ }^{\mathrm{e}}$ Competence Center Radiation Protection and Radiochemistry, Austrian Agency for Health and Food Safety, Spargelfeldstrasse 191, 1226 Vienna, Austria \\ ${ }^{\mathrm{f}}$ Deutscher Wetterdienst, Frankfurter Street 135, 63067 Offenbach, Germany \\ ${ }^{\mathrm{g}}$ GSF-National Center for Environment and Health, Institute of Radiation Protection 85764 Neuherberg, Germany \\ ${ }^{\mathrm{h}}$ Swiss Federal Office of Public Health, Environmental Radioactivity Section, c/o Physics Department University, 1700 Fribourg, Switzerland
}

\begin{abstract}
After the nuclear reactor accident of Chernobyl, the "Integrated Measurement and Information System (IMIS) for Monitoring the Environmental Radioactivity and Detecting Emissions from Nuclear Plants was implemented in Germany. IMIS is a nationwide comprehensive measuring system which permanently monitors the radioactivity in all important environment media in the whole federal territory. At approximately 40 sites, the activity concentration of radioactive substances is measured in air and precipitations. At least 14 of them are responsible for trace monitoring of radionuclides in the air. The legal bases of IMIS prescribe regular inter-laboratory comparison analyses in cooperation with the Physikalisch-Technische Bundesanstalt (PTB), with the use of reference materials prepared by the Federal Coordinating Laboratories. In order to fulfil this requirement in the field of trace survey measurements in ground-level air, the Federal Office for Radiation Protection ("Bundesamt für Strahlenschutz", BfS) and the PTB have conducted a comparison with real, dust-loaded reference filters in 2005. The comparison was organized within the framework of a cooperation of trace survey stations from Austria, Germany and Switzerland. The paper describes the preparation of the real, dust-loaded reference filters, the procedure for spiking the filters with the activity standard solution containing ${ }^{22} \mathrm{Na},{ }^{88} \mathrm{Y},{ }^{89} \mathrm{Sr},{ }^{90} \mathrm{Sr},{ }^{125} \mathrm{Sb},{ }^{133} \mathrm{Ba},{ }^{134} \mathrm{Cs}$, and ${ }^{241} \mathrm{Am}$. Some results are discussed and conclusions are given.
\end{abstract}

\section{Introduction}

After the accident at the Chernobyl nuclear power plant, the German Government passed the Federal Precautionary Radiation Protection Act "Strahlenschutzvorsorgegesetz" (BGB1, 2003) in December 1986. This Act prescribes an "Integriertes Mess- und Informationssystem", Integrated Measurement and Information System (IMIS) for monitoring environmental radioactivity and the radioactivity contained in foodstuffs. The Federal Coordinating Laboratories are requested to provide certified reference materials for

\footnotetext{
${ }^{*}$ Corresponding author. Tel.: + 49531592 6331; fax: + 495315926109

E-mail address: herbert.wershofen@ptb.de (H. Wershofen).
}

comparisons. The German environmental monitoring programme is described in the Bundesanzeiger (2006).

Within the scope of IMIS, "trace survey stations" for measurements of airborne radioactivity are in operation. The trace level defines activity concentrations of $<100 \mu \mathrm{Bq} / \mathrm{m}^{3}$. The tasks of trace survey stations are to monitor the existing levels of contamination and to detect any trends in order to establish the necessary baseline levels for a potential future contamination. Twelve trace survey stations are operated by the "Deutscher Wetterdienst" (DWD, German Meteorological Service) Offenbach and are distributed all over Germany. One more is operated in Freiburg by the BfS and another by PTB in Braunschweig. The European Union (EU) operates a "Sparse Network" 
for environmental radioactivity monitoring. Therefore, four German trace survey stations also report to the European Commission in order to fulfil the requirements of articles 35 and 36 of the EURATOM treaty.

The legal bases of IMIS prescribe regular inter-laboratory comparison analyses on reference materials. For this purpose, PTB provides a suitable activity standard solution to a Federal Coordinating Laboratory where the reference materials are prepared, and subsequently subsets of the reference material are distributed to the measuring laboratories. Besides the spiked reference materials, also non-spiked environmental material may be produced. One of the subsamples is randomly sent to PTB, where it also follows with the obligatory IMIS procedures.

In the field of trace survey measurements in ground-level air, the BfS and the PTB have conducted comparisons with real dust-loaded, large-area reference filters in 2003 in cooperation with trace survey stations in Austria, Germany, and Switzerland (Bieringer and Wershofen, 2005) and in 2005.

\section{Organization and operations}

Most of the parameters influencing air dust sampling (e.g. volume measurement, sample pre-treatment, radiochemical procedure, activity measurement) can be controlled by means of a quality management system (e.g. according to DIN EN ISO/IEC 17025, 2005). But the real weather conditions during sampling or the properties of the air dust sampler influencing the aerosol collection efficiency cannot be controlled so precisely. Normally, these parameters are the limiting factors for comparing measurement results from different sampling stations although they can be formally taken into account in an uncertainty budget. Hence, for the purpose of quality control it is more suitable to look at the total activity of a particular filter than at the activity concentrations measured in air.

The advantages of the method include

- achievement of traceability,

- freely selectable radionuclide composition depending only on availability,

- pre-adjustable activity level,

- analysing real samples,

- applying routine sample treatment,

- allowing for user-specific counting geometry,

- control of detector calibration,

- control of coincidence summation correction, and

- control of self-attenuation correction.

The radionuclides are selected according to the user requirements and the reference activities can be adjusted to environmental activity levels. Sometimes, the participants wish to receive a reference filter containing longer-lived, single $\gamma$-ray-emitting radionuclides to be used for routine control of the efficiency calibration. For that purpose, it is sufficient to spike a fresh filter. If self-attenuation needs to be taken into account, a dust-loaded filter should be spiked. In contrast, when radiochemical procedures are necessary in order to produce a radiochemically pure counting source (e.g. for isotopes of $\mathrm{Sr}, \mathrm{U}$, or $\mathrm{Pu}$ ), the spiked reference filter must inevitably be destroyed.

Disadvantages of the method are

- different filter materials,

- different filter dimensions,

- different air dust properties,

- artificial radionuclides in ambient air dust,

- different chemical properties of the radioelements,

- time-consuming and laborious spiking procedure, and

- a large number of control measurements for securing the reference activity.

In this comparison, five institutes participated. Their different filter types consisted of polypropylene, a mixture of glass fibres with cellulose fibres, or of glass fibres. The filter shapes were rectangular and the effective filter areas varied from 0.04 to $0.29 \mathrm{~m}^{2}$, resulting in between 1250 and 1600 droplets per filter. They were applied at a distance of between 5 and $10 \mathrm{~mm}$. In order to achieve the necessary homogeneity of the reference activity on the filter, it is not possible to go below a certain minimum number of droplets per filter.

The focus of this comparison was on coincidence summing correction, self-attenuation correction, radiochemistry for $\mathrm{Sr}$, and on the detector calibrations. The activity standard solution containing ${ }^{22} \mathrm{Na},{ }^{88} \mathrm{Y}$, ${ }^{89} \mathrm{Sr},{ }^{90} \mathrm{Sr},{ }^{125} \mathrm{Sb},{ }^{133} \mathrm{Ba},{ }^{134} \mathrm{Cs}$, and ${ }^{241} \mathrm{Am}$ was prepared and certified at PTB. The spiking of the filters and the quality control measurements were also performed at PTB. Table 1 presents the reference activities and the uncertainties.

Spiking was performed manually using an $x-y$ translation table which allows the dispenser to deposit sources at a number of finite positions in both directions. An automated system is under development. The ability to absorb water of the dust-loaded filters was checked prior to spiking and, in some cases, it was necessary to improve it with a detergent in order to prevent activity losses due to runoff. The filters were dried, carefully folded and sealed into polyethylene bags. All auxiliary materials (paper towels, gloves, clips, etc.) used for each reference filter were measured until the detection limit achieved was in the range of the uncertainty of the corresponding reference activity (usually about $1 \mathrm{~d}$ of counting was required). After no $\gamma$-activity of any target radionuclide was detected, the filters were shipped. The participants were asked to monitor the cover of their filter, as well as the auxiliary materials they had used for the preparation of their counting sources. Here too, no loss of activity was detectable.

The reporting date was about 6 months after shipment of the filters. The results were reported to BfS for compilation 
Table 1

The radionuclides and the reference activities $A_{r e f} \pm u\left(A_{r e f}\right)$ in $\mathrm{Bq}$ at reference time spiked to the real dust-loaded filters

\begin{tabular}{|c|c|c|c|c|c|c|}
\hline Radionuclide & Filter 1 & Filter 2 & Filter 3 & Filter 4 & Filter 5 & Filter 6 \\
\hline${ }^{22} \mathrm{Na}$ & $0.59 \pm 0.01$ & $0.55 \pm 0.01$ & $0.55 \pm 0.01$ & $0.58 \pm 0.01$ & $0.53 \pm 0.01$ & $0.76 \pm 0.02$ \\
\hline${ }^{88} \mathrm{Y}$ & $1.11 \pm 0.02$ & $1.03 \pm 0.02$ & $1.02 \pm 0.02$ & $1.09 \pm 0.02$ & $0.98 \pm 0.02$ & $1.42 \pm 0.03$ \\
\hline${ }^{89} \mathrm{Sr}^{\mathrm{a}}$ & $2.75 \pm 0.07$ & $2.56 \pm 0.07$ & $2.55 \pm 0.07$ & $2.73 \pm 0.07$ & $2.45 \pm 0.07$ & $3.56 \pm 0.11$ \\
\hline${ }^{90} \mathrm{Sr}^{\mathrm{b}}$ & $1.12 \pm 0.03$ & $1.04 \pm 0.03$ & $1.04 \pm 0.03$ & $1.11 \pm 0.03$ & $1.00 \pm 0.03$ & $1.44 \pm 0.04$ \\
\hline${ }^{125} \mathrm{Sb}$ & $0.46 \pm 0.01$ & $0.43 \pm 0.01$ & $0.42 \pm 0.01$ & $0.45 \pm 0.01$ & $0.41 \pm 0.01$ & $0.59 \pm 0.01$ \\
\hline${ }^{133} \mathrm{Ba}$ & $0.38 \pm 0.01$ & $0.35 \pm 0.01$ & $0.35 \pm 0.01$ & $0.37 \pm 0.01$ & $0.34 \pm 0.01$ & $0.49 \pm 0.01$ \\
\hline${ }^{134} \mathrm{Cs}$ & $0.54 \pm 0.01$ & $0.50 \pm 0.01$ & $0.50 \pm 0.01$ & $0.53 \pm 0.01$ & $0.48 \pm 0.01$ & $0.68 \pm 0.01$ \\
\hline${ }^{241} \mathrm{Am}$ & $0.58 \pm 0.01$ & $0.54 \pm 0.01$ & $0.53 \pm 0.01$ & $0.57 \pm 0.01$ & $0.51 \pm 0.01$ & $0.54 \pm 0.01$ \\
\hline
\end{tabular}

All uncertainties are the standard uncertainties in accordance with the IMIS convention.

${ }^{a}$ In order to assure reasonable counting statistics, even after a greater activity loss in the course of the radiochemical procedure, and to cover the decay of the short-lived ${ }^{89} \mathrm{Sr}$, the reference activities of ${ }^{89} \mathrm{Sr}$ were relatively high.

${ }^{\mathrm{b}} \mathrm{The}$ reference activities of ${ }^{90} \mathrm{Sr}$ were relatively high, because two potentially interfering contributions to the ${ }^{90} \mathrm{Sr}$ reference activity have to be taken into account:

(1) ${ }^{90} \mathrm{Sr}$ contained in ambient air dust and

(2) ${ }^{90} \mathrm{Sr}$ possibly contained as an impurity in the ${ }^{89} \mathrm{Sr}$ activity standard solution, caused by the production mode of the ${ }^{89} \mathrm{Sr}$. Therefore, the $A_{\text {ref }}{ }^{90} \mathrm{Sr}$ ) were chosen so that any potential contribution of impurities was covered by the $u\left(A_{\text {ref }}\right)$.

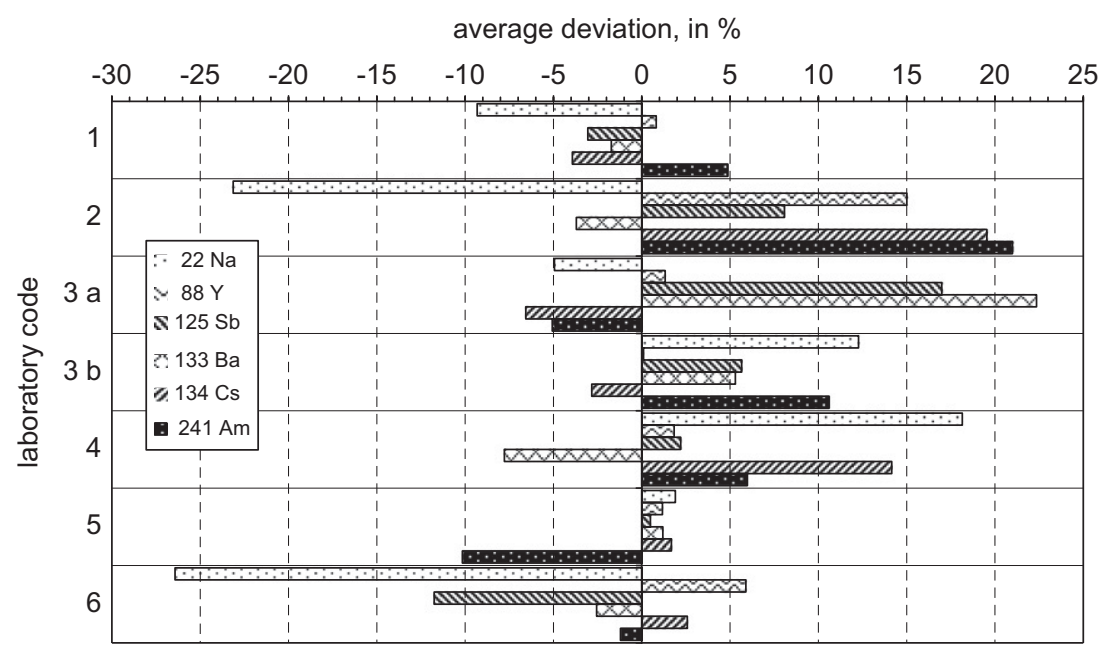

Fig. 1. The results of the $\gamma$-spectrometric measurements.

and evaluation; the comparison report was elaborated by BfS and PTB jointly.

\section{Results and discussion}

The participants (Austrian Agency for Health and Food Safety, BfS, DWD, GSF-National Center for Environment and Health, and PTB) were asked to report the results of the two measurements and the combined measurement uncertainties according to the ISO (GUM) Guide to the Expression of the Uncertainty in Measurement (ISO/ BIPM, 1995). However, not all participants had measured the $\mathrm{Sr}$ or $\mathrm{Y}$ counting sources twice and not in all cases a detailed uncertainty budget was reported for both the $\gamma$ - and the $\beta$-measurements. All uncertainties are the standard uncertainties in accordance with the IMIS convention (Bundesanzeiger, 2006).

One possible reason for the missing uncertainty budgets may be that some laboratories had difficulties in properly taking the coincidence summing correction and the selfattenuation correction properly into account. This is being investigated and a software programme "UncertRadio" is under development at the Federal Research Centre for Fisheries (Kanisch, 2007). It is planned to provide it to the IMIS community after its validation will be finished.

The results of the $\gamma$-ray-emitting radionuclides are presented in Fig. 1, those of ${ }^{90} \mathrm{Sr}$ in Fig. 2, and those of ${ }^{89} \mathrm{Sr}$ in Fig. 3. Missing data mean "no result reported" because not all participants have determined either ${ }^{90} \mathrm{Sr}$ $(\# 1)$ or ${ }^{89} \mathrm{Sr}(\# 1$ and $\# 4)$. There is one more $\gamma$-spectrometric measurement than reference filters, because one participant 


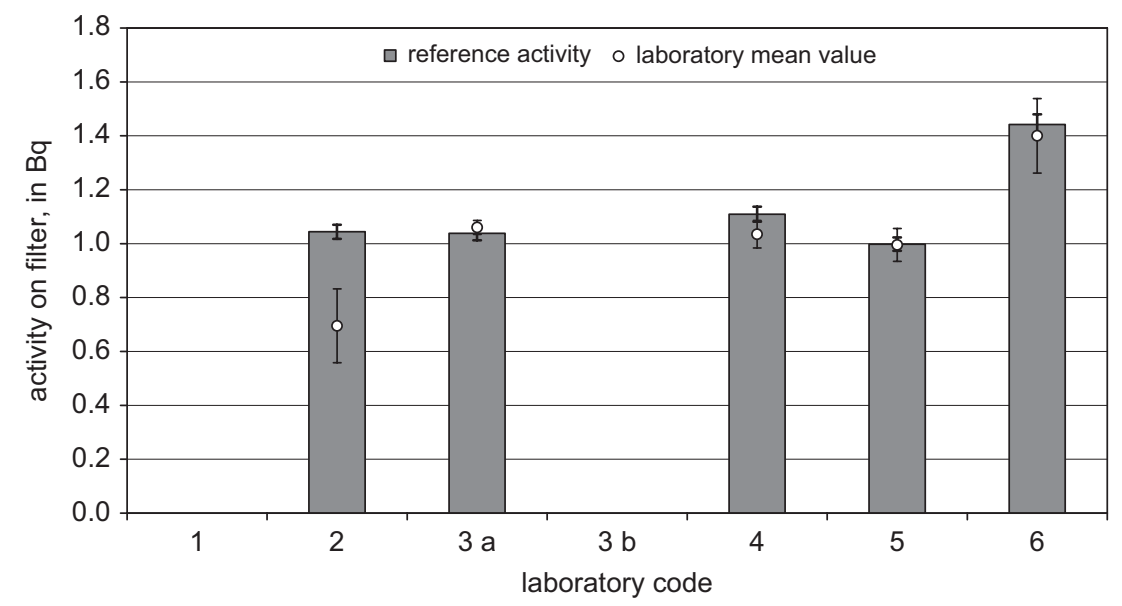

Fig. 2. The results of the ${ }^{90} \mathrm{Sr}$ measurements, including standard uncertainties of reference and lab mean values.

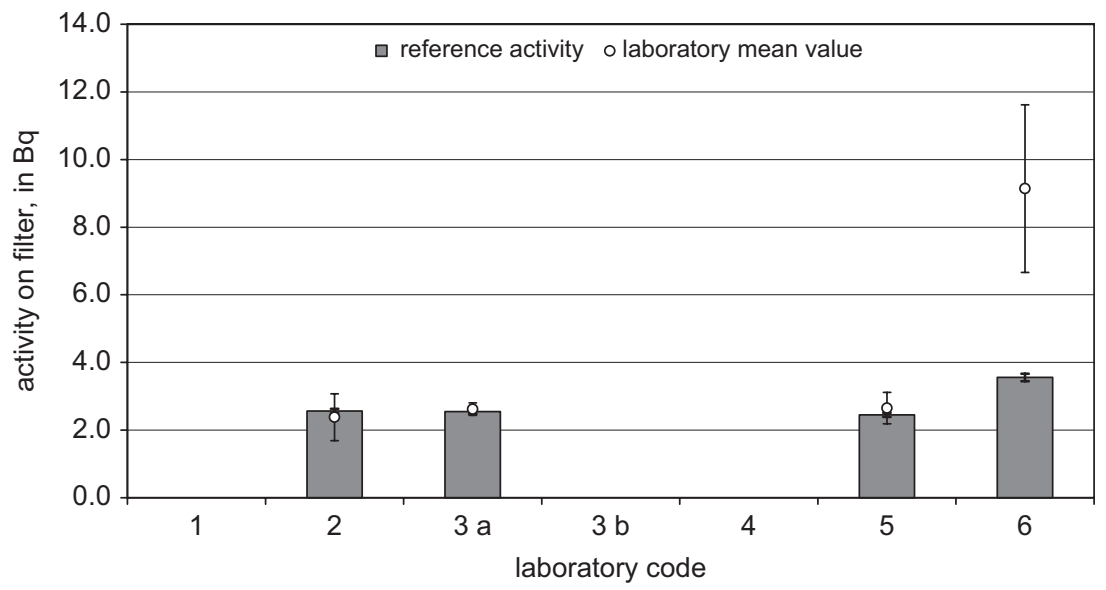

Fig. 3. The results of the ${ }^{89} \mathrm{Sr}$ measurements, including standard uncertainties of reference and lab mean values.

has measured in two different geometries ( $\# 3 \mathrm{a}$ and $\# 3 \mathrm{~b}$ ). The rapid measurement method ( $\# 3 b$ ) which is to be used for daily samples taken under emergency situations does not allow for uncertainties better than ca. $10 \%$ due to the differences in the activity distribution on the filter (contained in a $1 \mathrm{~L}$ Marinelli beaker) and in the liquid calibration source. For emergency situations, uncertainties in that range are acceptable. In order to achieve the lower limits of detection (typical: $0.03 \mu \mathrm{Bq}$ ${ }^{137} \mathrm{Cs} / \mathrm{m}^{3}$ ) necessary for environmental monitoring, in that laboratory the weekly air dust sample is washed and measured in a well-type detector (\#3a). In that geometry, coincidence summing is of much more importance than in other geometries.

The mean values of the results of the $\gamma$-spectrometric measurements (Fig. 1) deviate between $-27 \%$ and $+23 \%$. The larger deviations were observed for ${ }^{22} \mathrm{Na}$ and were caused by missing coincidence summing corrections. Coincidence summing of the $1274.5 \mathrm{keV}$ photon with a $511 \mathrm{keV}$ annihilation photon is neglected and the activity which is calculated from the $1274.5 \mathrm{keV}$ peak is too small.
In general, the results of the other measurements lie in the tolerable range of approx. $-15 \%$ to $+20 \%$. This is no surprise because these nuclides are well-known "calibration nuclides". But when looking at the details, one can see that there was some overcorrection: the ${ }^{133} \mathrm{Ba}$ result $\# 3$ deviates by approx. $22 \%$. This is the mean of two measurements performed with two different spectrometers resulting in individual deviations of approx. $11 \%$ and approx. $34 \%$. The reason for the larger difference is that the dimensions and properties of the well-type detectors available are not known precisely enough. As these parameters are essential for reliable coincidence summing corrections, one can very easily over- (or under-)correct for that. Especially when a large, X-ray-sensitive, $\mathrm{p}$-type detector is used so that also coincidence summing of $\gamma$-photons with X-rays has to be taken into account, a precise knowledge of the detector properties is indispensable.

With one exception, the results of the ${ }^{90} \mathrm{Sr}$ determinations are in good agreement with the reference activities. The outlier $\# 2$ was caused by a problem with the radiochemical yield tracer for ${ }^{90} \mathrm{Y}$ which was used. Today, 
a commercially available ICP-MS standard is used in that laboratory.

When looking at the results for ${ }^{89} \mathrm{Sr}$, one can see that the result of laboratory $\# 2$ is correct. This indicates that there was a problem with the calibration of the LSC-spectrometer for ${ }^{90} \mathrm{Sr}$, which emits $\beta$-particles of lower energy than ${ }^{89} \mathrm{Sr}$. The basic recommendation here is to cross-check the validity of a multi-nuclide calibration against the corresponding single-nuclide calibrations.

The result of laboratory $\# 6$ has a deviation of approx. $+150 \%$. Currently (September 2007), it is still open whether this is due to a calibration error or due to a contamination of the $\beta$-counting source with some $\beta$-particle emitter(s) insufficiently removed in the radiochemical procedure. The recommendation here is to carefully check the separation technique applied for robustness against interfering matrix effects.

\section{Conclusions and outlook}

From the monitoring point of view, the results of the comparison show that the measurements are reasonably precise for both routine environmental monitoring and emergency management practice. The results of the $\mathrm{Sr}$ measurements deviate in a wider range due to the wellknown problems arising from both the radiochemical procedures as well as from properly calibrating the measurement devices.

From the metrological point of view, more efforts seem to be necessary, in particular in order to improve the coincidence summing corrections for the "high-efficiency measurement geometries". The estimation of the measurement uncertainties should be performed consistently in accordance with an international standard in order to achieve a better comparability of the results.

For future comparisons, it is planned to extend the number of participants and to offer participation to more European trace survey stations. This will be possible when an automated spiking device is available.

\section{Acknowledgement}

We thank Mrs. Almuth Mitsch from the working group "Environmental Radioactivity" at PTB for her patience and accuracy in spiking the reference filters.

\section{References}

BGB1. I., 2003. Gesetz zum vorsorgenden Schutz der Bevölkerung gegen Strahlenbelastung (Strahlenschutzvorsorgegesetz-StrVG) vom 19. Dezember 1986 (BGBl. I S. 2610), zuletzt geändert durch Achte Zuständigkeitsanpassungsverordnung vom 25. November 2003, S. 2304, 2308 (in German).

Bieringer, J., Wershofen, H., 2005. Bestimmung des Radionuklidgehaltes im Luftstaub-Ringversuch Spurenanalyse 2003/2004, Report SW 3.5-1/2005. Bundesamt für Strahlenschutz, Willy-Brandt-Straße 5, 38226 Salzgitter, Germany (in German).

Bundesanzeiger Nr. 244, 2006. Allgemeine Verwaltungsvorschrift zum Integrierten Mess- und Informationssystem zur Überwachung der Radioaktivität in der Umwelt (IMIS) nach dem Strahlenschutzvorsorgegesetz (AVV-IMIS) vom 13.12.2006, S. 7413-7421 (in German).

DIN EN ISO/IEC 17025, 2005. General Requirements for the Competence of Testing and Calibration Laboratories. International Organization for Standardization, Geneva, Switzerland.

ISO/BIPM, 1995. Guide to the Expression of Uncertainty in Measurement. International Organization for Standardization, Geneva, Switzerland.

Kanisch, G., 2007. Federal Research Centre for Fisheries, Institute for Fishery Ecology. (Mr. G. Kanisch is the contact person for detailed information. E-mail: guenther.kanisch@vti.bund.de.) 